Results Samples from 14 patients (11 males, mean age 56 years) with the Cough Hypersensitivity Syndrome and 10 lung resection samples ( 6 males, mean age 68 years) were obtained. All the tissue samples stained for TRPA1. The TRPA1 stain was avidly taken up by the bronchial epithelium, smooth muscle bundles and nerve tissue.

Conclusion We have described the distribution of the TRPA1 ion channel in lung tissue. TRPA1 is agonised by several environmental irritants and endogenous mediators of inflammation. The presence of TRPA1 ion channel in bronchial mucosal nerves and epithelium suggests an important role in the cough reflex. The identification of these ion channels on smooth muscle could suggest a role in asthmatic inflammation as well.

\section{S142 InVESTIgATING PATTERNS IN 24 HOURS OF COUGHING}

doi:10.1136/thoraxjnl-2011-201054b.142

${ }^{1} \mathrm{R}$ J Dockry, ${ }^{1} \mathrm{~K}$ Sunger, ${ }^{2} \mathrm{P}$ Marsden, ${ }^{2} \mathrm{~A}$ Kelsall, ${ }^{2} \mathrm{~S}$ Decalmer, ${ }^{3} \mathrm{~W}$ Powley, ${ }^{3} \mathrm{R}$ Murdoch, ${ }^{1} \mathrm{~A}$ Woodcock, 'J A Smith. ${ }^{1}$ University of Manchester, Manchester, UK; ${ }^{2}$ University Hospital of South Manchester, Manchester, UK; ${ }^{3}$ GlaxoSmithKline, Discovery Medicine, Stevenage, UK

Introduction and Objectives Ambulatory $24 \mathrm{~h}$ cough monitoring is a relatively new technique, and so far few studies have investigated hourly variation in cough frequency. We analysed data from previous studies in three different disease groups to compare patterns throughout the day.

Methods We studied chronic cough (duration $>8$ weeks) $[n=70$, median age 58 yrs (IOR 49-64), 67.1\% female], acute cough in otherwise healthy volunteers (duration $<3$ weeks) $[\mathrm{n}=56$, median age 22 yrs (IOR 21-25.5), 62.5\% female] and physician diagnosed asthma [ $n=58$, median age 58.5 yrs (IOR $52-67.8$ ), 63.8\% female]. Subjects wore a cough monitor (Vitalojak) for $24 \mathrm{~h}$ and median numbers of coughs in each hour were manually counted and plotted against the actual time of day.

Results The asthmatic group had significantly lower total cough rates compared with the other groups (geometric mean 2.3 coughs/hr (SD \pm 3.3$)$; chronic cough 13.1 ( \pm 2.7$)$; acute cough 8.9 $( \pm 2.63) ; \mathrm{p}<0.001)$. Abstract S142 figure 1 shows the median coughs in each hour of the day (interquartile ranges). The chronic and acute cough groups show a very similar cough frequency and pattern, with two peaks during the daytime, despite substantial variability. The asthma group show a similar pattern but with much lower counts. For all diagnosis coughing practically ceases overnight.

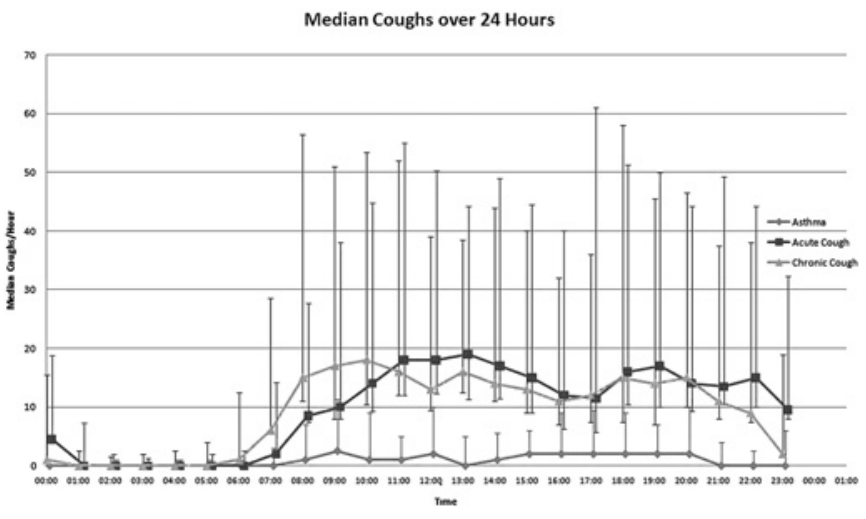

\section{Abstract S142 Figure 1}

Conclusions These data suggest that cough frequency in acute and chronic cough are remarkably similar, implying similar mechanisms may be driving coughing in these conditions, unlike in asthma where cough frequency is much lower. However all groups show similar fluctuations in hour to hour pattern of daytime cough and effective suppression of coughing during sleep, indicating behaviour has an important influence.

\section{S143 THE SENSATIONS PROVOKING COUGH: A QUALITATIVE INVESTIGATION}

doi:10.1136/thoraxjnl-2011-201054b.143

${ }^{1} \mathrm{~F}$ Solari, ${ }^{1} \mathrm{~K}$ Sunger, ${ }^{2} \mathrm{~J}$ Yorke, ${ }^{1} \mathrm{~A}$ Caress, ${ }^{1} \mathrm{~J} \mathrm{~A}$ Smith. ${ }^{1}$ The University of Manchester, University Hospital South Manchester, Manchester, UK; ${ }^{2}$ The University of Salford, School of Nursing and Midwifery, Greater Manchester, UK

Background Little is known about the sensations patients feel prior to or that provoke their cough.

Aims To identify, from the patient's perspective, key descriptors that relate to the sensations provoking cough in patients diagnosed with one of the following: idiopathic cough, asthma, chronic obstructive pulmonary disease (COPD), fibrotic interstitial lung disease (ILD) and cystic fibrosis.

Methods One-to-one, audio recorded, semi- structured interviews were conducted with 34 participants $(7$ idiopathic cough: age 40-69 years, 5 female, 6 asthma: age 42-65 years, 5 female, 7 COPD: age 52-86 years, 2 female, 7 ILD: age 58-79 years, 3 female, 7 cystic fibrosis: age 20-39 years, 2 female). Words and phrases relating to 1 of 8 top level codes, derived from the interview topic guide (triggers, sensation, sputum, emotion, location, frequency, time and relief) were extracted using simple manifest content analysis. These descriptions were then organised into similar themes. Relationships between the descriptions and themes both within and between the groups were explored.

Results All groups described the sensation of a "tickle" or an "irritation" that was felt most in the throat and upper chest. Cystic fibrosis and COPD participants most commonly described feeling a "need to clear" their airways. The ILD group mostly described a "dry", "tickly" sensation. The idiopathic cough and asthma groups used a very heterogeneous set of terms regarding the sensations provoking their cough. Common across the groups is a hypersensitive response to things that may trigger their urge to cough and all groups described coughing as relieving that urge. Emotionally the sensation is associated with negative emotions, however positive feelings were also described; by some who described feeling a need to clear their airways.

Conclusion The extracted list of descriptions has provided some insight into what the sensations provoking cough feel like from patient's perspective and suggest that the sensations felt relate to the pathology of the individual's diagnosis. The distinction between the need to clear and a sensation of a tickle or irritation could be important in understanding the mechanisms driving cough and may be important for the development of targeted interventions.

\section{S144 ACUTE COUGH: A LONGITUDINAL OBSERVATIONAL STUDY}

doi:10.1136/thoraxjnl-2011-201054b.144

${ }^{1} \mathrm{~K}$ K Lee, ${ }^{2} \mathrm{~S}$ Matos, ${ }^{3} \mathrm{D}$ H Evans, I I Pavord, ${ }^{4} \mathrm{~S}$ S Birring. ${ }^{1}$ Division of Asthma, Allergy and Lung Biology, King's College London, London, UK; ${ }^{2}$ Institute of Electronics and Telematics Engineering (IEETA), University of Aveiro, Portugal; ${ }^{3}$ Department of Medical Physics, Leicester Royal Infirmary, Leicester, UK; ${ }^{4}$ Department of Respiratory Medicine, Institute for Lung Health, Glenfield Hospital, Leicester, UK

Introduction Acute cough is a common illness with more than 12 million episodes each year in the UK. More than $£ 100$ million is 